\title{
Enhanced Framework to Evaluate Performance \& Functionality of Web Applications
}

\author{
M.Prasanna kumar \\ M.Tech (cse) computer science \\ Vellore institute of technology \\ Vellore.
}

\author{
K.Mohan \\ Asst.proffsor(senior), SCSE \\ Vellore institute of technology \\ Vellore.
}

\begin{abstract}
A system's efficiency is always determined by the performance like how the system responses to an input and what/ when /where the expected output is obtained, As performance and functionality are the most important features in a web application. Due to lack of multi-directional analysis, most of the software's are not exceeding the bench mark of perfection both in case of performance and also functionality. So this paper gives a framework, Writing Automation rules to check the functionality, fixing the bench mark and checking the performance of the application and creating statistical reports based on the bench marks is the core prototype of this paper. In our paper we create a web application to check the functionality using a technology Quick Test Professional (QTP). So manual flaws can be easily avoided. Then the load test is checked using Loader Runner. Using this load test technology, the application is simulated with multiple users and checked for the performance of the application in terms of different stress levels(hits/s, response time, throughput, errors/s).Added to that, this paper is extended to check the threshold of the system, how much amount of consumption of energy in system for the particular running application. A detailed report will be provided on functionality and stable performance (average response time) of the web applications.
\end{abstract}

\section{Key Words}

Software testing, functional testing, performance testing, QTP, load Runner, test case.

\section{INTRODUCTION}

The explosive growth of applications and web services has changed the present scenario in exchanging the information, in government, corporate or educational and research institutes. The productivity and operational efficiencies have been increased to manifold with the development of sophisticated but simple applications. With such development, the responsibility on the developer's side is unlimited. Although the applications would be provided with easy to use features, at the same time these applications must also be able to handle large number of concurrent users. In such a situation, as most of the businesses are conducted through web, it is very important and crucial to get the application be tested. In software development life cycle, testing is one of the most important features and probably the least understood part [2]. The growth of web services with web applications made business transactions much simpler.

\section{RELATED WORKS}

Providing an optimized performance benchmarked system is always a challenging area in the market. Still, lot of software providers are aggressively trying to make this happen using some of the software testing tools. Due to lack of multi directional analysis most of the software's are not exceeding the bench mark of perfection both in the performance and also functionality. Lack of proper testing the application leads to connection failure, memory leakage, Increase in response time, system error[1].

Testing is done for performance using Load Runner $[1,4]$ by choosing .Netas framework and evaluated the performance of .Net technology for developing web applications. In the same way [2] Osama Hamed and NedalKafri investigated on performance evaluation of web applications using load Runner but they tested results on basis of platforms .Net and Java, they reported that .Net platform provides stable results.

From authors J. Križani, A. Grguri, M. Mošmondor, P. Lazarevski research provided report of existing performance evaluation tools by testing them with AJAX based environment, after comparing all testing tools they said Grinder Analyzer provides optimum performance for AJAX based applications [5]. The performance analysis provides the end-user to know the intention of the developed application is completed as its gives the efficiency of the developed application [6].The performance is also evaluated using work load characterization from customer behavior model graphs and author Politi [8]came with a tool named Walty. Then evaluation methods (i.e) parameters increased but performance analysis is done by Load runner only $[9,4]$.

Many research have been done to evaluate performance of the web application as they provide as bridge to communicate business information with clients. So it is mandatory field to evaluate performance in multi-directional way to meet the intention of application and also to avoid potential cost invested for development of web applications.

\section{DESIGN METHODOLOGY}

Our system is designed with .Net as framework using Patent Registration as web application; initially we check the functionalities of the application by specifying the automation rules by inserting the check points using QTP (quick test professional) technology.

As functionality testing is done in presence of the client, who needs the application so during that process tester will provide demonstration of the developed application in that process client can realize whether the appearance and functionality is 
developed according to his need, if not tester can configure the requirement from client, then after testing using QTP, bug report is generated and sent to the developer for further modification. The bug report contains test summary so developer can understand where to do the changes.

After completion of functionality we check the performance of the application by using the load runner, here the application is simulated against number of virtual users, under these parameters (throughput, response time, hits/sec, error/sec) from the analysis we can find the stable response time of the application. Generated results are represented in graphical flowcharts, which gives the stable performance of the developed web application. Adding the threshold check point in this application is also an challenging criteria for this system.

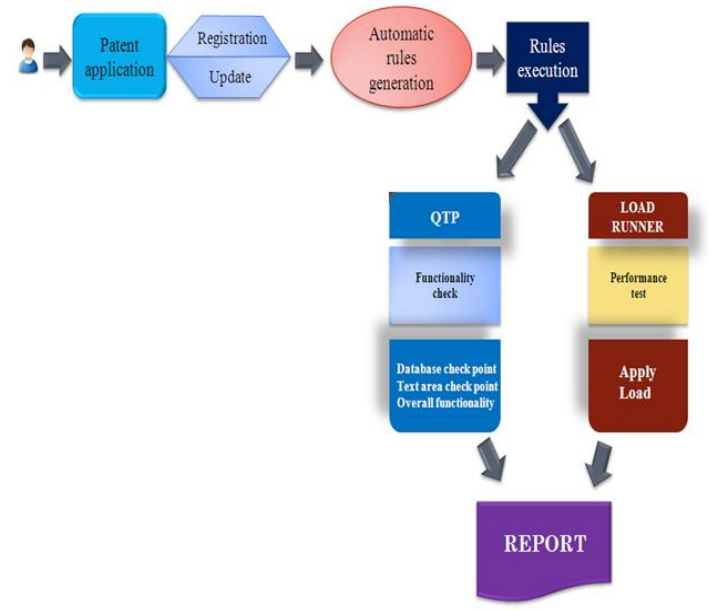

\subsection{System design}

\section{FUNCTIONALITY EXPLANATION}

\subsection{Patent application}

In our paper we have created an web application for registering patents, which includes registration under various areas of domain and priorities, claims, number of pages, year of publication. If the user is not an register, he can register using NEW USER REGISTRATION FORM, following fields like View, search, edit, option can be accessed by authenticated user only. This application is created as gateway to demonstrate our idea, but this concept can be worked in any web applications in the market, with limitations.

\subsection{Automation Rules}

Here QTP is used to create automation rules defining how the application should react. We will be customizing the tool to check for functionality under following parameters, text, textarea, bitmap, database and overall functional flow of the application.

\subsection{QTP Automation rules generation}

\subsubsection{Description of checkpoints}

Standard checkpoint: here functionality testing is done to check the properties of enable and disable options of cursers according the user requirement.

Database checkpoint: checks for the table content and stored values that are correctly retrieved when required. Also checks on updates made.
Text checkpoint: this testing gives properties of buttons.

Text area checkpoint: here the testing is done to check, after which word next word should appear.

Bitmap checkpoint: here various images are compared and checked for the dynamic properties of image.

\subsection{QTP RULES EXECUTION}

Once the rules were framed, the application will be executed to check the functionality of the code. Mostly, the application and functionalities will checked in the system where there is huge enhancements and possibility of impact on the other modules. This behaves like an enhancement of functionalities.

\subsection{APPLYING LOAD}

Here using Load runner we will apply the load on the application to check the performance and stress capacity of the application. We are simulating the response time for one user and in parallel we will manipulate the numerous users accessing the same application. We use the concept of virtual users, we can change and configure the time of entry into application (time/s).

\subsection{PERFORMANCE EVALUATION}

we calculate the steady performance of the application in normal usage (in minimal usage), by that we can find the steady time of the application, by the same way applying load say $10,30,50,70$ users to the application and by simulating users with difference in time/s of entry into the application. We can determine the steady response of the application also the peak response time. Finally we can report that this application has stable response time at this number of user, when it goes beyond this range, the response time is not stable.

\subsubsection{Functionality testing}

In This testing we are going to check the functionality of the application. There are four test cases available in this such as Links, Forms, Database, and Cookies. It is one of the challenging criteria, since now no paper has said about this testing. It works similar to the real time industry testing environment.

\subsubsection{Links}

In this test case we consider the four types of links such as internal link, external link, mail link and broken link.

In theinternal link,we check whether the internal link of the system is correctly linked or not. The link is within the system.

In the external link, when link is between the two applications. We check whether it directs to the external link or not.

In themail link, we check the database whether the system take the values from the database or not.

In the broken link $\boldsymbol{k}_{\text {we }}$ check whether the system links to the broken or to the error page.

\subsubsection{Forms}

In this form type of testing we check the whether the system gives the error message for the wrong input or not. We also 
check whether the system gives the error for not giving the input for the mandatory field. Hence, we write the test case for the mandatory field and the wrong input.

\subsubsection{Database}

In this, we check whether the system's data thatis updated to the database .Here we check database for Modify, Update, Delete options.

\subsection{PERFORMANCE TESTING \\ 4.7.1. Load}

In this we are going to check the maximum load which the system can able to maintain. Here we use the LOAD RUNNER to check the maximum load for the system. In this application features are provided to include number of VIRTUAL USERS, and also time/s to enter into application as user. From the load runner results, we can find steady time and peak time of the application. Results can be compared on the basis of the parameters (responsetime, hits/s, throughput, error/s) along with number of users. So the performance of the application can be determined.

\section{RESULTS AND DISCUSSIONS}

After the completion of working with application against the testing technology, The QTP gives the functionality of the web application in means of testcases and test summary report, which contains necessary changes (bug report) which can be sent to the developer for further enhancement. Then LOAD RUNNER gives the statistical graphs regarding the response time, hits/sec, throughput, about the various load given to application, Which contains the performance report of the web application. The performance and functionality can be tested against any existing web applications to make live demonstration, but there are some limitations while handling load runner, because of generating multiple users from same IP address, the server will block the IP address of the machine.

\section{SNAPSHOTS OF RESULTS}

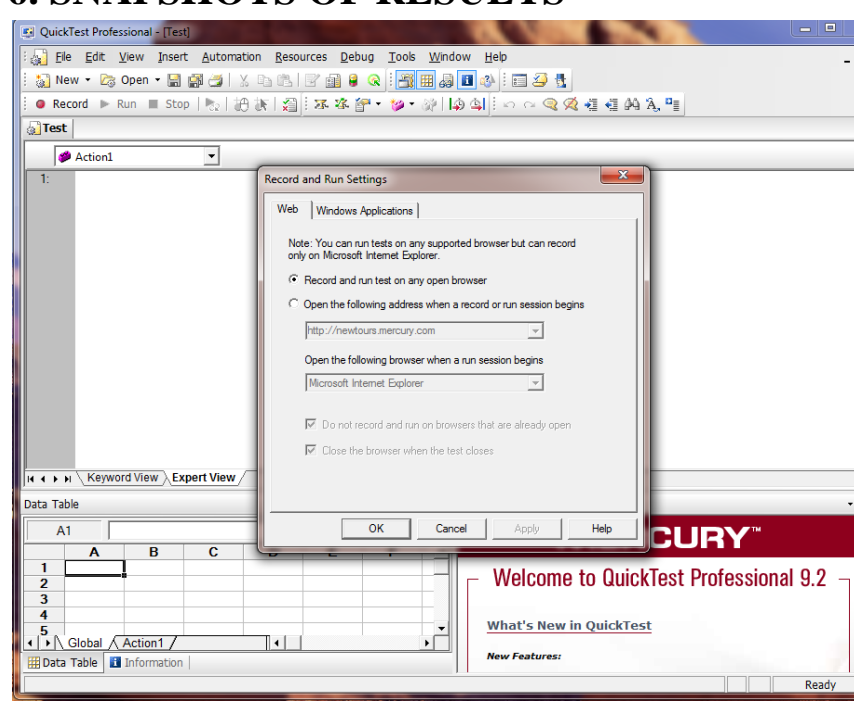

6.1.QTP tool
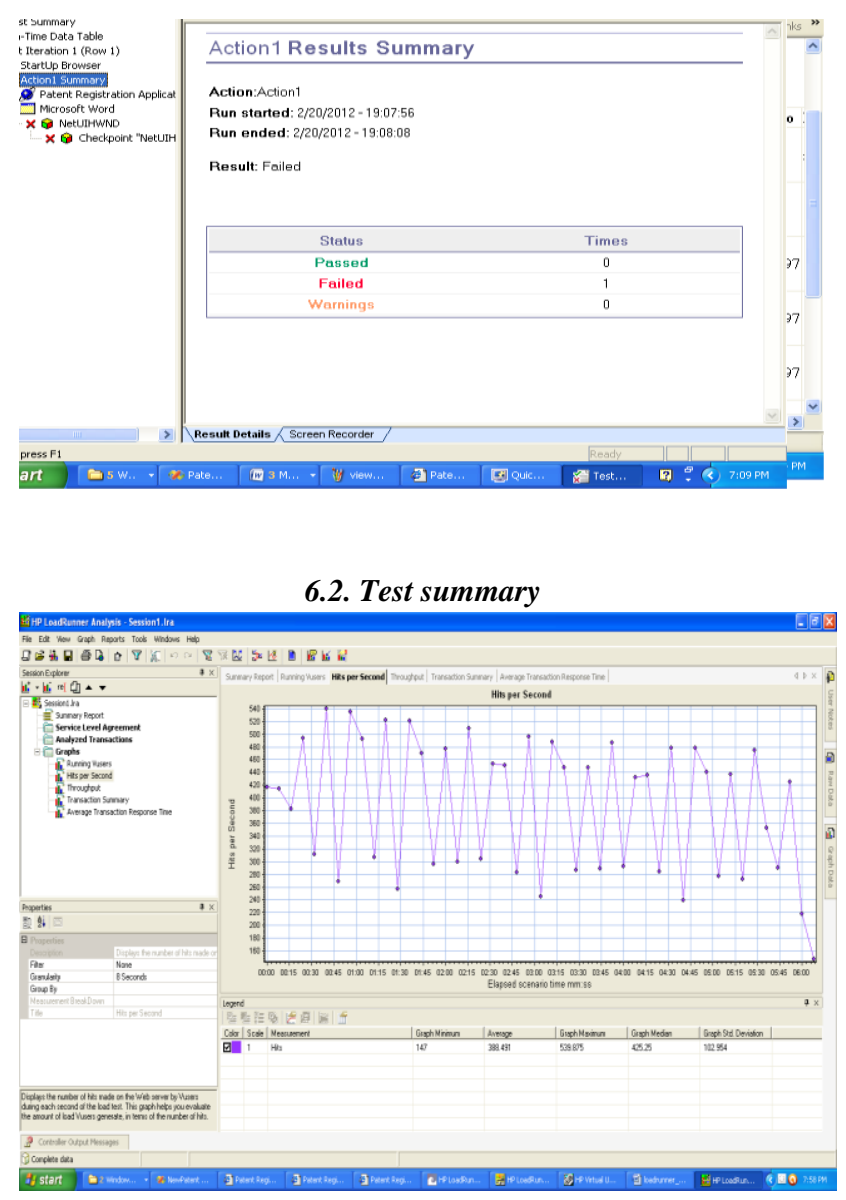

6.3 Load runner

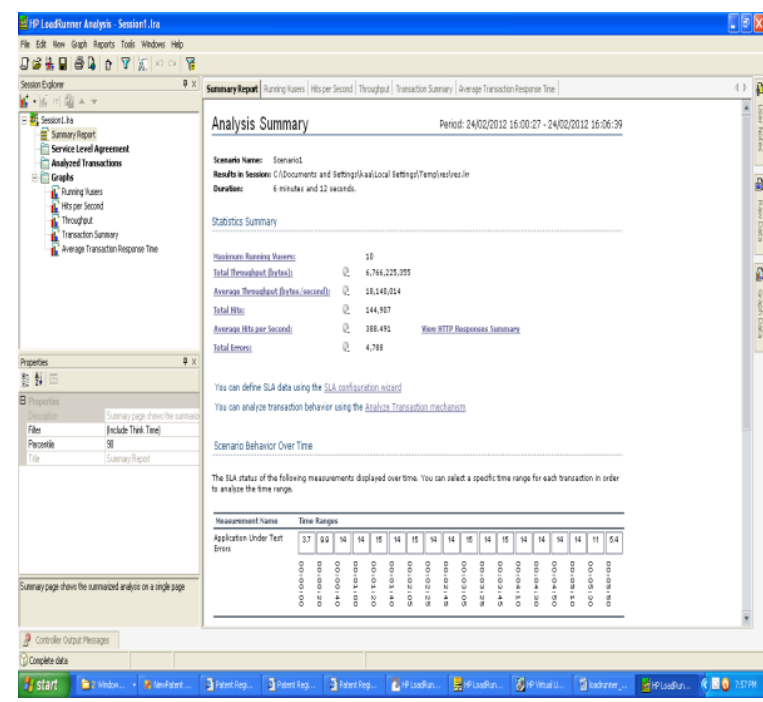

\subsection{Performance report}

\section{CONCLUSION}

Our paper gives the deep knowledge of an developed web application, end-user satisfaction is most important task in project development. From the above testing on application the client can extract the performance, functionality and stability of the application. So that user can divide his resources accordingly to give quick response. The client will be satisfied of purchasing 
a cost effective project. We have an idea of implementing the concept of threshold as our future work.

\section{REFERENCES}

[1] M.Kalita1 T.Bezboruah "Investigation on performance testing and evaluation of PReWebD: a .NET technique for implementing web application", (IET software) pp, 357365 on 2011.

[2] Osama Hamed1 and NedalKafri "Performance Testing for Web Based Application Architectures (.NET vs. Java EE)”, (IEEE) 2009.

[3] Taolun Chai Zibing and Wang/Jiaxin Wang , "Automated Universal Testing and Tutoring System for Web Application” (IEEE) 2009.

[4] Puyunming and Xumingna , "Load Testing for Web Applications" on 2009

[5] J.Križani, A.Grguri, M.Mošmondor, P.Lazarevski ,"Load testing and performance monitoring tools in use with AJAX based web Applications” MIPRO 2010.
[6] DileepBhandarkar,"Performance Analysis in the Real World of On Line Services", Conference speech in Microsoft 2009 .

[7] YujiaGe andYuanyanGu ."Scenario Based Performance Analysis of Distributed Web Applications", ACM 2010.

[8] G. Ruffo, R. Schifanella, and M. Sereno R. Politi , "WALTy: A tool for Evaluating Web Application Performance" (IEEE) 2004.

[9] Cheng-hui Huang, and Huo Yan Chen , "A Tool to Support Automated Testing for Web ApplicationScenario" (First international conference on cybernetics system )2006.

[10] Yanyan Lu, Haiyan Wu, Yingxue Wang , "Web Application Performance Analysis Based on Comprehensive Load Testing" ICWMMN 2006.

[11] Qinglin Wu and Yan Wang , "Performance Testing and Optimization of J2EE-based Web Applications" ( Second International Workshop on Education Technology and Computer Science)2010. 